\title{
A novel approach for multiple criteria group decision making problem with unknown criteria weight information
}

\author{
V. Sireesha and K. Himabindu \\ Department of Applied Mathematics, GITAM \\ Visakhapatnam, India \\ e-mails: vsirisha80@gmail.com, himabindu.chelamkuri@gmail.com
}

Received: 8 October $2017 \quad$ Revised: 22 November $2017 \quad$ Accepted: 30 November 2017

\begin{abstract}
In this paper, an approach to multiple criteria group decision making (MCGDM) problem in which the criteria weights are unknown is proposed. The normal distribution is applied to criteria values to find the criteria weights when the decision maker weights, criteria values are taken in the form of interval valued intuitionistic fuzzy trapezoidal numbers (IVIFTNs). The effectiveness of the developed approach is verified by giving an illustrative example.

Keywords: Multiple criteria group decision making, Interval-valued intuitionistic fuzzy trapezoidal numbers, Unknown criteria weight, Interval-valued intuitionistic fuzzy trapezoidal weighted averaging operator.
\end{abstract}

AMS Classification: 03E72.

\section{Introduction}

The concept of fuzzy sets (FS) was introduced by Zadeh [12] in 1965. Later fuzzy sets were generalized by Atanassov [1], called the intuitionistic fuzzy sets (IFS). The IFS includes membership function and non-membership functions which takes the exact values [0, 1]. But it is more effective to represent membership or non-membership functions in interval form rather than exact numbers for application to real world problems. Atanassov and Gargov [2] gave the concept of interval valued intuitionistic fuzzy sets (IVIFSs) [9]. The domain in IFSs and IVIFSs is a discrete set; therefore their membership degrees and the non-membership degrees can only express fuzzy concept in terms of "excellent" or "good". To overcome this limitation, Shu et al. [5] defined intuitionistic fuzzy triangular numbers for which domain is a consecutive set. Then 
the extension of ITFNs that is the intuitionistic fuzzy trapezoidal numbers (IFTNs) given by Wang [8]. Later on Wan [7] introduced the concepts of interval valued intuitionistic fuzzy trapezoidal numbers (IVIFTNs), in which both membership and non-membership are in interval forms. So, it is more effective to study the MCGDM under the view of IVIFTNs.

In decision making problems, it is very important that to aggregate the overall information. The weighted averaging aggregation is a very common technique among all procedures of MCGDM. There are many aggregation operators and ordered weighted averaging operators are proposed by researchers to aggregate FNs, IFNs, IVIFNs and IVIFTNs. Guiwu et al. [3] introduced some operational laws of interval intuitionistic fuzzy trapezoidal numbers and applied interval intuitionistic trapezoidal fuzzy ordered weighted geometric (IITFOWG) and interval valued intuitionistic trapezoidal fuzzy hybrid geometric (IITFHG) operators for decision making. Later on, interval valued intuitionistic trapezoidal fuzzy weighted geometric operator (IVITFWG), interval valued intuitionistic trapezoidal fuzzy ordered weighted operator (IVITFOWG), and interval valued intuitionistic trapezoidal fuzzy weighted hybrid operators (IVITFWH) and their properties introduced by $\mathrm{Wu}$ and Liu [9]. If weights of the criteria are completely unknown, there are criteria-independent and criteria-dependent approaches to find the weights. $\mathrm{Xu}$ [10] proposed normal distribution based method which includes the number of criteria but independent on the values of criteria. Hence, in this paper we proposed a criteriadependent approach to find the criteria weights using normal distribution. Subsequently, the interval-valued intuitionistic fuzzy trapezoidal weighted averaging operator (IVIFTWA) is used to find the best alternative among the given.

\section{Preliminaries}

In this section, we briefly introduce some basic concepts related to interval valued intuitionistic trapezoidal fuzzy numbers and their arithmetic operations.

Definition 1 [1]: Intuitionistic Fuzzy Set. An intuitionistic fuzzy set over universe of discourse $X$ is of the form: $\mathrm{A}=\left\{\left\langle x, \mu_{\tilde{A}}(x), v_{\tilde{A}}(x) \mid x \in X\right\rangle\right\}$, where $\mu_{A}$ denotes membership function, and $v_{A}$ denotes non-membership function, with the condition $0 \leq \mu_{\tilde{A}}(x)+v_{\tilde{A}}(x) \leq 1, \mu_{\tilde{A}}(x), v_{\tilde{A}}(x)$ $\in[0,1]$ for all $x \in X$.

Definition 2 [2]: Interval valued intuitionistic fuzzy set. An interval-valued intuitionistic fuzzy set in $\mathrm{A}$ over $\mathrm{X}$ is an object having the form:

$$
A=\left\{\left\langle x,\left[\mu_{\tilde{A}}^{L}(x), \mu_{\tilde{A}}^{U}(x)\right],\left[v_{\tilde{A}}^{L}(x), v_{\tilde{A}}^{U}(x)\right]\right\rangle|x \in X\rangle\right\},
$$

where $\mu_{\tilde{A}}^{L}, \mu_{\tilde{A}}^{U}, v_{\tilde{A}}^{L}, v_{\tilde{A}}^{U}: X \rightarrow[0,1], \mu_{\tilde{A}}^{L} \leq \mu_{\tilde{A}}^{U}, \quad v_{\tilde{A}}^{L} \leq v_{\tilde{A}}^{U}$

Definition 3 [11]: IVIFNs Score Function. Let $\tilde{A}=\left(\left[\mu_{\tilde{A}}^{L}, \mu_{\tilde{A}}^{U}\right],\left[v_{\tilde{A}}^{L}, v_{\tilde{A}}^{U}\right]\right)$ be an interval-valued intuitionistic fuzzy number. The score function of $\tilde{A}, S_{X}(\tilde{A})$ is represented as,

$$
S_{X}(\tilde{A})=\frac{\mu_{\tilde{A}}^{L}+\mu_{\tilde{A}}^{U}-v_{\tilde{A}}^{L}-v_{\tilde{A}}^{U}}{2}, S_{X}(\tilde{A}) \in[-1,1] .
$$


Definition 4 [11]: IVIFNs Accuracy Function. Let $\tilde{A}=\left(\left[\mu_{\tilde{A}}^{L}, \mu_{\tilde{A}}^{U}\right],\left[v_{\tilde{A}}^{L}, v_{\tilde{A}}^{U}\right]\right)$ be an intervalvalued intuitionistic fuzzy number. The accuracy function of $\tilde{A}, H_{X}(\tilde{A})$ is represented as;

$$
H_{X}(\tilde{A})=\frac{\mu_{\tilde{A}}^{L}+\mu_{\tilde{A}}^{U}+v_{\tilde{A}}^{L}+v_{\tilde{A}}^{U}}{2}, H_{X}(\tilde{A}) \in[0,1] .
$$

Definition 5 [8]: Interval Valued Intuitionistic Fuzzy Trapezoidal Sets (IVIFTS). Let $\tilde{A}$ be an interval-valued intuitionistic fuzzy trapezoidal set (IVIFTS), its interval-valued membership function is:

$$
\mu_{\tilde{A}}^{U}(x)=\left\{\begin{array}{cc}
\frac{x-a}{b-a} \mu_{\tilde{A}}^{U}, & a \leq x<b ; \\
\mu_{\tilde{A}}^{U}, & b \leq x \leq c ; \\
\frac{d-x}{d-c} \mu_{\tilde{A}}^{U}, & c<x \leq d ; \\
0, & \text { others. }
\end{array}\right.
$$

and

$$
\mu_{\tilde{A}}^{L}(x)=\left\{\begin{array}{cc}
\frac{x-a}{b-a} \mu_{\tilde{A}}^{L}, & a \leq x<b \\
\mu_{\tilde{A}}^{L}, & b \leq x \leq c ; \\
\frac{d-x}{d-c} \mu_{\tilde{A}}^{L}, & c<x \leq d ; \\
0, & \text { others. }
\end{array}\right.
$$

Its interval-valued non-membership function is:

$$
v_{\tilde{A}}^{U}(x)=\left\{\begin{array}{cc}
\frac{b-x+v_{\tilde{A}}^{U}(x-a)}{b-a}, & a \leq x<b ; \\
v_{\tilde{A}}^{U}, & b \leq x \leq c ; \\
\frac{x-c+v_{\tilde{A}}^{U}(d-x)}{d-c}, & c<x \leq d ; \\
0, & \text { others. }
\end{array}\right.
$$

and

$$
v_{\tilde{A}}^{L}(x)=\left\{\begin{array}{cc}
\frac{b-x+v_{\tilde{A}}^{L}(x-a)}{b-a}, & a \leq x<b ; \\
v_{\tilde{A}}^{L}, & b \leq x \leq c ; \\
\frac{x-c+v_{\tilde{A}}^{L}(d-x)}{d-c}, & c<x \leq d ; \\
0, & \text { others }
\end{array}\right.
$$

where $0 \leq \mu_{\tilde{A}}^{L} \leq \mu_{\tilde{A}}^{U} \leq 1 ; 0 \leq v_{\tilde{A}}^{L} \leq v_{\widetilde{A}}^{U} \leq 1 ; 0 \leq \mu_{\tilde{A}}^{U}+v_{\widetilde{A}}^{U} \leq 1 ; 0 \leq \mu_{\tilde{A}}^{L}+v_{\tilde{A}}^{L} \leq 1 ; a, b, c, d \in R$. Then $\tilde{A}=\left([a, b, c, d] ;\left[\mu_{\tilde{A}}^{L}, \mu_{\tilde{A}}^{U}\right] ;\left[v_{\tilde{A}}^{L}, v_{\tilde{A}}^{U}\right]\right)$ is called an interval-valued intuitionistic fuzzy trapezoidal set (IVIFTS). 


\section{Definition 6 [8]: Arithmetic Operation law of IVITFS. Let}

$$
\tilde{A}_{1}=\left(\left[a_{1}, b_{1}, c_{1}, d_{1}\right] ;\left[\mu_{\tilde{A}_{1}}^{L}, \mu_{\tilde{A}_{1}}^{U}\right] ;\left[v_{\tilde{A}_{1}}^{L}, v_{\tilde{A}_{1}}^{U}\right]\right)
$$

and

$$
\tilde{A}_{2}=\left(\left[a_{2}, b_{2}, c_{2}, d_{2}\right] ;\left[\mu_{\tilde{A}_{2}}^{L}, \mu_{\tilde{A}_{2}}^{U}\right] ;\left[v_{\tilde{A}_{2}}^{L}, v_{\tilde{A}_{2}}^{U}\right]\right)
$$

be two interval-valued intuitionistic fuzzy trapezoidal numbers, then

$$
\begin{aligned}
\tilde{A}_{1} \oplus \tilde{A}_{2}= & \left(\left[a_{1}+a_{2}, b_{1}+b_{2}, c_{1}+c_{2}, d_{1}+d_{2}\right] ;\left[\mu_{\tilde{A}_{1}}^{L}+\mu_{\tilde{A}_{2}}^{L}-\mu_{\tilde{A}_{1}}^{L} \mu_{\tilde{A}_{2}}^{L}, \mu_{\tilde{A}_{1}}^{U}+\mu_{\tilde{A}_{2}}^{U}-\mu_{\tilde{A}_{1}}^{U} \mu_{\tilde{A}_{2}}^{U}\right] ;\right. \\
& {\left.\left[v_{\tilde{A}_{1}}^{L} v_{\tilde{A}_{2}}^{L}, v_{\tilde{A}_{1}}^{U} v_{\tilde{A}_{2}}^{U}\right]\right) }
\end{aligned}
$$

$\tilde{A}_{1} \otimes \tilde{A}_{2}=\left(\left[a_{1} a_{2}, b_{1} b_{2}, c_{1} c_{2}, d_{1} d_{2}\right] ;\left[\mu_{\tilde{A}_{1}}^{L} \mu_{\tilde{A}_{2}}^{L}, \mu_{\tilde{A}_{1}}^{U} \mu_{\tilde{A}_{2}}^{U}\right]\right.$

$$
\left.\left[v_{\tilde{A}_{1}}^{L}+v_{\tilde{A}_{2}}^{L}-v_{\tilde{A}_{1}}^{L} v_{\tilde{A}_{2}}^{L}, v_{\tilde{A}_{1}}^{U}+v_{\tilde{A}_{2}}^{U}-v_{\tilde{A}_{1}}^{U} v_{\tilde{A}_{2}}^{U}\right]\right)
$$

$$
\lambda \tilde{A}_{1}=\left(\left[\lambda a_{1}, \lambda b_{1}, \lambda c_{1}, \lambda d_{1}\right] ;\left[1-\left(1-\mu_{\tilde{A}_{1}}^{L}\right)^{\lambda}, 1-\left(1-\mu_{\tilde{A}_{1}}^{U}\right)^{\lambda}\right],\left[\left(v_{\tilde{A}_{1}}^{L}\right)^{\lambda},\left(v_{\tilde{A}_{1}}^{U}\right)^{\lambda}\right]\right)
$$

Definition 7 [6]: Value index of IVITFN. Based on the values of membership function and nonmembership function, the value index of IVITFN $\tilde{A}=\left([a, b, c, d] ;\left[\mu_{\tilde{A}}^{L}, \mu_{\tilde{A}}^{U}\right] ;\left[v_{\tilde{A}}^{L}, v_{\tilde{A}}^{U}\right]\right)$ is defined as

$$
V(\tilde{A})=\frac{a+2(b+c)+d}{12}\left(1+S_{x}(\tilde{A})-H_{x}(\tilde{A})\right) .
$$

Definition 8 [6]: Ambiguity index of IVITFN. Based on the ambiguities of membership function and non-membership function, the ambiguity index of IVITFN $\tilde{A}=\left([a, b, c, d] ;\left[\mu_{\tilde{A}}^{L}, \mu_{\tilde{A}}^{U}\right] ;\left[v_{\tilde{A}}^{L}, v_{\tilde{A}}^{U}\right]\right)$ is defined as,

$$
A(\tilde{A})=\frac{(d-a)-2(b-c)}{6}\left(1+S_{x}(\tilde{A})-H_{x}(\tilde{A})\right)
$$

Based on the value index function $V(\tilde{A})$ and the ambiguity index function $A(\tilde{A})$, the following ranking procedure is proposed: For two interval-valued intuitionistic fuzzy trapezoidal numbers

$$
\tilde{A}_{1}=\left(\left[a_{1}, b_{1}, c_{1}, d_{1}\right] ;\left[\mu_{\tilde{A}_{1}}^{L}, \mu_{\tilde{A}_{1}}^{U}\right] ;\left[v_{\tilde{A}_{1}}^{L}, v_{\tilde{A}_{1}}^{U}\right]\right)
$$

and

$$
\tilde{A}_{2}=\left(\left[a_{2}, b_{2}, c_{2}, d_{2}\right] ;\left[\mu_{\tilde{A}_{2}}^{L}, \mu_{\tilde{A}_{2}}^{U}\right] ;\left[v_{\tilde{A}_{2}}^{L}, v_{\tilde{A}_{2}}^{U}\right]\right)
$$

1) If $V\left(\tilde{A}_{1}\right)<V\left(\tilde{A}_{2}\right)$, then $\tilde{A}_{1}<\tilde{A}_{2}$

2) If $V\left(\tilde{A}_{1}\right)>V\left(\tilde{A}_{2}\right)$, then $\tilde{A}_{1}>\tilde{A}_{2}$

3) If $V\left(\tilde{A}_{1}\right)=V\left(\tilde{A}_{2}\right)$ then find $A\left(\tilde{A}_{1}\right)$ and $A\left(\tilde{A}_{2}\right)$

i) If $A\left(\tilde{A}_{1}\right)<A\left(\tilde{A}_{2}\right)$ then $\tilde{A}_{1}<\tilde{A}_{2}$

ii) If $A\left(\tilde{A}_{1}\right)>A\left(\tilde{A}_{2}\right)$ then $\tilde{A}_{1}>\tilde{A}_{2}$

iii) If $A\left(\tilde{A}_{1}\right)=A\left(\tilde{A}_{2}\right)$ then $\tilde{A}_{1}=\tilde{A}_{2}$ 
Definition 9: IVIFTWA Operator. Let $\Omega$ be the set of interval-valued intuitionistic fuzzy trapezoidal number and let $\tilde{A}_{i} \in \Omega, i=1,2,3, \ldots n$. An interval-valued intuitionistic fuzzy trapezoidal weighted averaging operator, IVIFTWA: $\Omega^{n} \rightarrow \Omega$ is defined as

$$
\operatorname{IVIFTWA} A_{\tilde{w}}\left(\tilde{A}_{1}, \tilde{A}_{2}, \ldots, \tilde{A}_{n}\right)=\tilde{w}_{1} \otimes \tilde{A}_{1} \oplus \tilde{w}_{2} \otimes \tilde{A}_{2} \oplus \ldots \oplus \tilde{w}_{n} \otimes \tilde{A}_{n}
$$

where $\tilde{w}=\left(\tilde{w}_{1}, \tilde{w}_{2}, \ldots \tilde{w}_{n}\right)^{T}$ be the fuzzy weight vector of $\tilde{A}_{i}, i=1,2,3, \ldots n, \tilde{w}_{i} \geq \tilde{0}, \sum_{i=1}^{n} \tilde{w}_{i}=1$ with the weights given by

$$
\begin{aligned}
& \tilde{w}_{1}=\left(\left[l_{1}, m_{1}, n_{1}, o_{1}\right] ;\left[\mu_{\tilde{w}_{1}}^{L}, \mu_{\tilde{w}_{1}}^{U}\right] ;\left[v_{\tilde{w}_{1}}^{L}, v_{\tilde{w}_{1}}^{U}\right]\right), \\
& \tilde{w}_{2}=\left(\left[l_{2}, m_{2}, n_{2}, o_{2}\right] ;\left[\mu_{\tilde{w}_{2}}^{L}, \mu_{\tilde{w}_{2}}^{U}\right] ;\left[v_{\tilde{w}_{2}}^{L}, v_{\tilde{w}_{2}}^{U}\right]\right), \\
& \ldots \\
& \tilde{w}_{n}=\left(\left[l_{n}, m_{n}, n_{n}, o_{n}\right] ;\left[\mu_{\tilde{w}_{n}}^{L}, \mu_{\tilde{w}_{n}}^{U}\right] ;\left[v_{\tilde{w}_{n}}^{L}, v_{\tilde{w}_{n}}^{U}\right]\right) .
\end{aligned}
$$

\section{Proposed method to find the best alternative when the criteria weights are unknown in advance}

In multicriteria decision making problems, different weights will be assigned to criteria depending on the problem. Sometimes, the criteria weight is completely unknown in advance. There are criteria-independent and criteria-dependent approaches to find the weights criteria [4]. In this section, the method to find the weight of the criteria and best alternative is given in step wise. In the present approach, the given criteria values are used together with normal distribution to obtain the criteria weights, given in Definition 10. As the approach is criteria dependent, it can relieve the influence of unfair arguments on the decision by assigning low weights to those false and biased.

Definition 10. The weights of the criteria is defined as

$$
\tilde{W}_{C_{i}}=\frac{1}{\sqrt{2 \pi \sum_{i=1}^{n} A\left(C_{i}\right)}} e^{-\left[\frac{\left[V\left(C_{i}\right)-\sum_{i=1}^{n} V\left(C_{i}\right)\right]^{2}}{2 \sum_{i=1}^{n} A\left(C_{i}\right)}\right]}, i=1,2,3,4, \ldots, n
$$

where $V\left(C_{i}\right)$ and $A\left(C_{i}\right)$ is respectively the value index and ambiguity index of the criteria $i$. 
Step 1: Consider the experts weighting vector $\tilde{W}_{D}$. Normalize the experts weighting vectors using Definition 7. Then we obtain the normalized experts weighting vector $N \tilde{W}_{D}$.

Step 2: Apply IVITFWAA operator for assessments of decision makers on criteria with the normalized experts weighting vectors to obtain a decision matrix.

If $N \tilde{W}_{D_{1}}, N \tilde{W}_{D_{2}}, N \tilde{W}_{D_{3}}, \ldots, N \tilde{W}_{D_{l}}$ are normalized experts weighting vectors and let $\tilde{\alpha}_{i j}^{l}=$ $\left(\left[a_{i j}, b_{i j}, c_{i j}, d_{i j}\right] ;\left[\mu_{\tilde{\alpha}_{i j}}^{L}, \mu_{\tilde{\alpha}_{i j}}^{U}\right] ;\left[v_{\tilde{\alpha}_{i j}}^{L}, v_{\tilde{\alpha}_{i j}}^{U}\right]\right)$ represent the performance of alternative $A_{j}$ with respect to criterion $C_{i}$ by the expert $D_{l},(j=1,2,3, \ldots, m, i=1,2,3, \ldots . n$, $l=1,2,3, \ldots, k)$.

Then the decision matrix elements are obtained as follows

$$
\begin{aligned}
& \tilde{d}_{11}=N \tilde{W}_{D_{1}} \otimes \tilde{\alpha}_{11}^{1} \oplus N \tilde{W}_{D_{2}} \otimes \tilde{\alpha}_{11}^{2} \oplus \ldots N \tilde{W}_{D_{l}} \otimes \tilde{\alpha}_{11}^{l} \\
& \tilde{d}_{12}=N \tilde{W}_{D_{1}} \otimes \tilde{\alpha}_{21}^{1} \oplus N \tilde{W}_{D_{2}} \otimes \tilde{\alpha}_{21}^{2} \oplus \ldots N \tilde{W}_{D_{l}} \otimes \tilde{\alpha}_{21}^{l} \ldots \\
& \tilde{d}_{1 m}=N \tilde{W}_{D_{1}} \otimes \tilde{\alpha}_{m 1}^{1} \oplus N \tilde{W}_{D_{2}} \otimes \tilde{\alpha}_{m 1}^{2} \oplus \ldots N \tilde{W}_{D_{l}} \otimes \tilde{\alpha}_{m 1}^{l} \\
& \tilde{d}_{21}=N \tilde{W}_{D_{1}} \otimes \tilde{\alpha}_{12}^{1} \oplus N \tilde{W}_{D_{2}} \otimes \tilde{\alpha}_{12}^{2} \oplus \ldots N \tilde{W}_{D_{n}} \otimes \tilde{\alpha}_{12}^{l} \\
& \tilde{d}_{22}=N \tilde{W}_{D_{1}} \otimes \tilde{\alpha}_{22}^{1} \oplus N \tilde{W}_{D_{2}} \otimes \tilde{\alpha}_{22}^{2} \oplus \ldots N \tilde{W}_{D_{l}} \otimes \tilde{\alpha}_{22}^{l}, \\
& \tilde{d}_{2 m}=N \tilde{W}_{D_{1}} \otimes \tilde{\alpha}_{m 2}^{1} \oplus N \tilde{W}_{D_{2}} \otimes \tilde{\alpha}_{m 2}^{2} \oplus \ldots N \tilde{W}_{D_{l}} \otimes \tilde{\alpha}_{m 2}^{l} \ldots \\
& \tilde{d}_{n 1}=N \tilde{W}_{D_{1}} \otimes \tilde{\alpha}_{1 n}^{1} \oplus N \tilde{W}_{D_{2}} \otimes \tilde{\alpha}_{1 n}^{2} \oplus \ldots N \tilde{W}_{D_{l}} \otimes \tilde{\alpha}_{1 n}^{l} \\
& \tilde{d}_{n 2}=N \tilde{W}_{D_{1}} \otimes \tilde{\alpha}_{2 n}^{1} \oplus N \tilde{W}_{D_{2}} \otimes \tilde{\alpha}_{2 n}^{2} \oplus \ldots N \tilde{W}_{D_{l}} \otimes \tilde{\alpha}_{2 n}^{l}, \ldots \\
& \tilde{d}_{n m}=N \tilde{W}_{D_{1}} \otimes \tilde{\alpha}_{m n}^{1} \oplus N \tilde{W}_{D_{2}} \otimes \tilde{\alpha}_{m n}^{2} \oplus \ldots N \tilde{W}_{D_{l}} \otimes \tilde{\alpha}_{m n}^{l}
\end{aligned}
$$

Step 3: Obtain the aggregated values for each criteria from decision matrix by

For $C_{1}$, Aggregated value $=\frac{1}{l}\left(\tilde{d}_{11} \oplus \tilde{d}_{12} \oplus \ldots \oplus \tilde{d}_{1 m}\right)$

(' $l$ ' represents the number of experts and $l=1,2,3, \ldots, k$ )

For $C_{2}$, Aggregated value $=\frac{1}{l}\left(\tilde{d}_{21} \oplus \tilde{d}_{22} \oplus \ldots \oplus \tilde{d}_{2 m}\right)$

For $C_{n}$, Aggregated value $=\frac{1}{l}\left(\tilde{d}_{n 1} \oplus \tilde{d}_{n 2} \oplus \ldots \oplus \tilde{d}_{n m}\right)$

Step 4: Calculate the corresponding value index and ambiguity index for the obtained aggregated values of the criteria in Step 3. Then apply the proposed definition for criteria weights and normalize them to get $\sum_{i=1}^{n} \tilde{W}_{C_{n}}=1$. Now we get the values of $\tilde{W}_{C_{1}}, \tilde{W}_{C_{2}}, \ldots, \tilde{W}_{C_{n}}$.

Step 5: Apply the IVITFWA operator with the criteria weights obtained in Step 4 and the decision matrix obtained in Step 2 . Then we get the collective overall preference values $\tilde{t}_{j}$ of each alternative $A_{j}, j=1,2,3, \ldots, m$.

Step 6: Calculate the corresponding value index for $\tilde{t}_{j}$ that is $V\left(\tilde{t}_{j}\right)$ obtained from Step 5 and rank the best alternative using the proposed ranking method. 


\subsection{Numerical example}

In this section, the proposed method is applied to find the best green supplier for a food company presented by $\mathrm{Wu}$ and Liu [4]. After pre-evaluation, three suppliers (alternatives) are selected for further evaluation. These suppliers are evaluated according to four criteria: Product quality $\left(C_{1}\right)$, Technology capability $\left(C_{2}\right)$, Pollution control $\left(C_{3}\right)$ and Environmental management $\left(C_{4}\right)$ by three decision makers namely $D M_{1}, D M_{2}, D M_{3}$. The weighting vector of the decision maker and criteria weights are respectively given by

$$
\tilde{W}_{\left\{D_{1}, D_{2}, D_{3}\right\}}=\left\{\begin{array}{c}
([0.1,0.15,0.20, .025]:[0.4,0.5],[0.3,0.4]), \\
([0.1,0.2,0.3,0.4] ;[0.4,0.6],[0.2,0.4]), \\
([0.3,0.4,0.5,0.6]:[0.3,0.5],[0.1,0.2])
\end{array}\right\}
$$

The assessments of three suppliers by three decision makers based on each criterion are given respectively in Table 1, Table 2 and Table 3.

Table 1: Assessment by $D M_{1}$

\begin{tabular}{|c|c|c|c|}
\hline \multirow{2}{*}{ 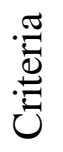 } & \multicolumn{3}{|c|}{ Suppliers } \\
\hline & $A_{1}$ & $A_{2}$ & $A_{3}$ \\
\hline$C_{1}$ & $([0.2,0.3,0.4,0.5] ;[0.3,0.6],[0.1,0.3])$ & $([0.1,0.3,0.4,0.5] ;[0.5,0.6],[0.2,0.4])$ & $([0.3,0.5,0.6,0.7] ;[0.3,0.4],[0.2,0.3])$ \\
\hline$C_{2}$ & $([0.4,0.6,0.7,0.8] ;[0.4,0.7],[0.1,0.2])$ & $([0.2,0.4,0.5,0.8] ;[0.4,0.5],[0.3,0.4])$ & $([0.1,0.3,0.4,0.5] ;[0.4,0.6],[0.1,0.2])$ \\
\hline$C_{3}$ & $([0.3,0.5,0.6,0.8] ;[0.3,0.4],[0.2,0.3])$ & $([0.4,0.6,0.8,1.0] ;[0.4,0.6],[0.2,0.4])$ & $([0.2,0.3,0.4,0.5] ;[0.4,0.5],[0.3,0.4])$ \\
\hline$C_{4}$ & $([0.1,0.3,0.4,0.6] ;[0.6,0.7],[0.2,0.3])$ & $([0.3,0.5,0.6,0.7] ;[0.3,0.5],[0.1,0.4])$ & $([0.1,0.2,0.3,0.5] ;[0.2,0.5],[0.1,0.4])$ \\
\hline
\end{tabular}

Table 2: Assessment by $D M_{2}$

\begin{tabular}{|c|c|c|c|}
\hline \multirow{2}{*}{ 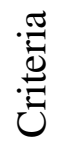 } & \multicolumn{3}{|c|}{ Suppliers } \\
\hline & $A_{1}$ & $A_{2}$ & $A_{3}$ \\
\hline$C_{1}$ & $([0.6,0.7,0.8,0.9] ;[0.6,0.7],[0.1,0.2])$ & $([0.5,0.6,0.7,0.8] ;[0.5,0.6],[0.3,0.4])$ & $([0.7,0.8,0.9,1.0] ;[0.5,0.8],[0.1,0.2])$ \\
\hline$C_{2}$ & $([0.1,0.2,0.4,0.5] ;[0.3,0.5],[0.2,0.4])$ & $([0.4,0.5,0.6,0.8] ;[0.4,0.6],[0.2,0.3])$ & $([0.3,0.4,0.5,0.6] ;[0.5,0.6],[0.2,0.4])$ \\
\hline$C_{3}$ & $([0.3,0.4,0.5,0.6] ;[0.5,0.6],[0.3,0.4])$ & $([0.1,0.2,0.3,0.5] ;[0.4,0.6],[0.2,0.4])$ & $([0.2,0.3,0.4,0.5] ;[0.7,0.8],[0.1,0.2])$ \\
\hline$C_{4}$ & $([0.1,0.3,0.4,0.5] ;[0.5,0.7],[0.2,0.3])$ & $([0.3,0.5,0.6,0.8] ;[0.3,0.4],[0.1,0.2])$ & $([0.2,0.3,0.4,0.5] ;[0.4,0.7],[0.2,0.3])$ \\
\hline
\end{tabular}

Table 3: Assessment by $D M_{3}$

\begin{tabular}{|c|c|c|c|}
\hline \multirow{2}{*}{ 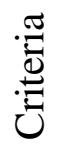 } & \multicolumn{3}{|c|}{ Suppliers } \\
\hline & $A_{1}$ & $A_{2}$ & $A_{3}$ \\
\hline$C_{1}$ & $([0.4,0.5,0.6,0.7] ;[0.4,0.5],[0.3,0.4])$ & $([0.5,0.6,0.7,0.9] ;[0.3,0.6],[0.1,0.2])$ & $([0.3,0.4,0.5,0.6] ;[0.5,0.6],[0.1,0.4])$ \\
\hline$C_{2}$ & $([0.5,0.6,0.7,0.9] ;[0.2,0.4],[0.1,0.2])$ & $([0.4,0.5,0.7,0.8] ;[0.4,0.5],[0.3,0.4])$ & $([0.2,0.3,0.4,0.5] ;[0.4,0.5],[0.2,0.5])$ \\
\hline$C_{3}$ & $([0.4,0.5,0.6,0.7] ;[0.7,0.8],[0.1,0.2])$ & $([0.2,0.3,0.4,0.5] ;[0.6,0.8],[0.1,0.2])$ & $([0.2,0.4,0.5,0.6] ;[0.5,0.6],[0.2,0.4])$ \\
\hline$C_{4}$ & $([0.5,0.6,0.7,0.8] ;[0.5,0.6],[0.2,0.4])$ & $([0.6,0.7,0.8,0.9] ;[0.3,0.4],[0.1,0.2])$ & $([0.7,0.8,0.9,1.0] ;[0.4,0.5],[0.3,0.5])$ \\
\hline
\end{tabular}




\section{Step 1: Normalization of decision makers' weights}

Experts weight is normalized using Eq. 3 and is given below

$$
N \tilde{W}_{\left\{D_{1}, D_{2}, D_{3}\right\}}=\left\{\begin{array}{l}
([0.08,0.15,0.27,0.50] ;[0.4,0.5],[0.3,0.4]), \\
([0.08,0.20,0.40,0.80] ;[0.4,0.6],[0.2,0.4]), \\
([0.24,0.41,0.67,1.20] ;[0.3,0.5],[0.1,0.2])
\end{array}\right\}
$$

Step 2: Calculation of the decision matrix

Table 4: Calculation of the decision matrix

\begin{tabular}{|c|c|c|c|}
\hline \multirow{2}{*}{ 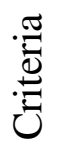 } & \multicolumn{3}{|c|}{ Suppliers } \\
\hline & $A_{1}$ & $A_{2}$ & $A_{3}$ \\
\hline$C_{1}$ & $\begin{array}{c}([0.16,0.39,0.83,1.81] ; \\
[0.42,0.69],[0.03,0.15])\end{array}$ & $\begin{array}{l}([0.16,0.41,0.85,1.97] \\
[0.41,0.68],[0.03,0.14])\end{array}$ & $\begin{array}{c}([0.15,0.39,0.85,1.87] \\
[0.40,0.70],[0.02,0.15])\end{array}$ \\
\hline$C_{2}$ & $\begin{array}{c}([0.16,0.37,0.81,1.88] ; \\
[0.31,0.63],[0.02,0.11])\end{array}$ & $\begin{array}{c}([0.14,0.36,0.84,2.0) \\
[0.37,0.64],[0.06,0.19])\end{array}$ & $\begin{array}{c}([0.08,0.24,0.57,1.33] \\
[0.41,0.66],[0.03,0.19])\end{array}$ \\
\hline$C_{3}$ & $\begin{array}{c}([0.14,0.36,0.76,1.72] ; \\
[0.44,0.69],[0.03,0.13])\end{array}$ & $\begin{array}{c}([0.08,0.25,0.61,1.50] \\
[0.42,0.73],[0.03,0.14])\end{array}$ & $\begin{array}{l}([0.08,0.26,0.60,1.37] \\
[0.48,0.72],[0.03,0.17])\end{array}$ \\
\hline$C_{4}$ & $\begin{array}{c}([0.13,0.33,0.73,1.66] ; \\
[0.48,0.73],[0.04,0.17])\end{array}$ & $\begin{array}{l}([0.19,0.46,0.939,2.07] \\
[0.29,0.54],[0.01,0.11])\end{array}$ & $\begin{array}{c}([0.19,0.41,0.84,1.85] ; \\
[0.31,0.67],[0.04,0.22])\end{array}$ \\
\hline
\end{tabular}

The aggregated value for $C_{1}=([0.16,0.39,0.83,1.86] ;[0.40,0.69],[0.03,0.15])$

The aggregated value for $C_{2}=([0.12,0.32,0.73,1.71] ;[0.36,0.64],[0.04,0.16])$

The aggregated value for $C_{3}=([0.11,0.29,0.65,1.51] ;[0.44,0.71],[0.03,0.15])$

The aggregated value for $C_{4}=([0.17,0.39,0.83,1.84] ;[0.36,0.65],[0.03,0.17])$

Step 3: Calculation of Value and Ambiguity indices, and Criteria weights

Value index:

$$
V\left(C_{1}\right)=0.303436 \quad V\left(C_{2}\right)=0.261455 \quad V\left(C_{3}\right)=0.236299 \quad V\left(C_{4}\right)=0.295944
$$

Ambiguity index:

$$
A\left(C_{1}\right)=0.350183 \quad A\left(C_{2}\right)=0.317878 \quad A\left(C_{3}\right)=0.287622 \quad A\left(C_{4}\right)=0.335054
$$

\section{Criteria weights:}

For $\tilde{W}_{C_{1}}$ :

$$
\tilde{W}_{C_{1}}=\frac{1}{\sqrt{2 \pi \sum_{i=1}^{n} A\left(C_{i}\right)}} e^{-\frac{\left[V\left(C_{1}\right)-\sum_{i=1}^{n} V\left(C_{i}\right)\right]^{2}}{2 \sum_{i=1}^{n} A\left(C_{i}\right)}} \quad, i=1,2,3,4
$$




$$
\begin{aligned}
\tilde{W}_{C_{1}} & =\frac{1}{\sqrt{2 \pi(1.290737)}} e^{-\frac{[0.303436-1.097134]^{2}}{2(1.290737)}} \\
& =0.2751156659
\end{aligned}
$$

Similarly

$$
\tilde{W}\left(C_{2}\right)=0.2679185735 \quad \tilde{W}\left(C_{3}\right)=0.2635256957 \quad \tilde{W}\left(C_{4}\right)=0.2738421911
$$

Normalized weights are

$$
\tilde{W}\left(C_{1}\right)=0.26 \quad \tilde{W}\left(C_{2}\right)=0.25 \quad \tilde{W}\left(C_{3}\right)=0.24 \quad \tilde{W}\left(C_{4}\right)=0.25
$$

\section{Step 5:}

By applying IVITFWA operator on the decision matrix from Step 2 with the normalized criteria weights obtained in step 4 we get the collective overall preference values as follows

$$
\begin{aligned}
& \tilde{t}_{1}=([0.15,0.37,0.79,1.77] ;[0.42,0.69],[0.03,0.14]) \\
& \tilde{t}_{2}=([0.15,0.38,0.82,1.89] ;[0.38,0.66],[0.03,0.15]) \\
& \tilde{t}_{3}=([0.13,0.33,0.73,1.61] ;[0.40,0.69],[0.03,0.18])
\end{aligned}
$$

\section{Step 6:}

Value index are

$$
V\left(\tilde{t}_{1}\right)=0.288391 \quad V\left(\tilde{t}_{2}\right)=0.300608 \quad V\left(\tilde{t}_{3}\right)=0.248925
$$

By ranking, we get $A_{2}>A_{1}>A_{3}$. Hence $A_{2}$ is the best alternative.

The result obtained by proposed method agrees with the $\mathrm{Wu}$ and Liu [9]. Moreover, the present approach orders the alternatives strictly when compared to [9].

\section{Acknowledgements}

The authors would like to thank Editor-in-chief and anonymous referees whose valuable suggestions and comments helped in improving the quality and presentation of the paper.

\section{References}

[1] Atanassov, K. (1986) Intuitionistic fuzzy sets, Fuzzy Sets and Systems, 20(1), 87-96.

[2] Atanassov, K., \& Gargov, G. (1989) Interval-valued intuitionistic fuzzy sets, Fuzzy Sets and Systems, 31(3), 343-349. 
[3] Guiwu, W., Xiaofei, Z., \& Hongjun, W. (2012) An approach to multiple attribute decision making with interval intuitionistic trapezoidal fuzzy information, Technological and Economic Development of Economy, 18 (2), 317-330.

[4] Qi, X.-W., Liang, C.-Y., \& Zhang, J. (2013) Some generalized dependent aggregation operators with interval-valued intuitionistic fuzzy information and their application to exploitation investment evaluation, Journal of Applied Mathematics, Volume 2013, Article ID 705159, 24 pages, http.//dx.doi.org/10.1155/2013/705159.

[5] Shu, M. H., Cheng, C. H., \& Chan, J. R. (2006) Using intuitionistic fuzzy sets for fault tree analysis on printed circuit board assembly, Microelectronics Reliability, 46, 2139-2148.

[6] Sireesha, V., \& Himabindu, K. (2016) An ELECTRE approach for multicriteria interval valued intuitionistic trapezoidal fuzzy group decision making problems, Advances in Fuzzy Systems, 10.1155/2016/1956303.

[7] Wan, S. P. (2011) Multi-attribute decision making method based on interval-valued intuitionistic trapezoidal fuzzy number, Control and Decision, 26(6), 857-860.

[8] Wang, J. Q. (2008) Overview on fuzzy multi-criteria decision making approach, Control and Decision, 23(6), 601-606.

[9] Wu, J., \& Liu, Y. (2013) An approach for multiple attribute group decision making problem with interval valued intuitionistic trapezoidal fuzzy numbers, Computers \& Industrial Engineering, 66, 311-324.

[10] Xu, Z.-S. (2005). An overview of methods for determining OWA weights. International Journal of Intelligent Systems, 20, 843-865.

[11] Xu, Z.-S. \& Chen, J. (2007) An approach to group decision making based on intervalvalued intuitionistic judgment matrices, System Engineering - Theory \& Practice, 27, 126-133.

[12] Zadeh, L. A. (1965) Fuzzy sets, Information and Control, 8, 338-353. 